\title{
Recurrence of borderline papillary serous tumors: a modern dilemma
}

Received: 23 March 2005 / Accepted: 23 August 2005 / Published online: 12 January 2006

(C) Springer-Verlag Berlin / Heidelberg 2006

\begin{abstract}
When defining tumors originating from the epithelial surface of the ovary, a distinction between benign and malignant is made based on clinical and pathologic features. This distinction allows clinicians to make difficult decisions as to which treatment will allow the best possible prognosis for patients with aggressive lesions, while avoiding unnecessary operations for those who are not likely to benefit from surgery. We would like to present a patient who was found in the operating room setting to have an extensive recurrence of papillary serous tumor of the ovary. Her original operative diagnosis was that of stage IC papillary serous ovarian carcinoma. The tumor re-occurred as a ventral hernia and during abdominal exploration she was found to have extensive disease. Although the survivability of borderline ovarian tumors has been well documented, their recurrence and sites of involvement are not easily defined. Additional studies are needed to further categorize serous borderline tumors and to treat them appropriately.
\end{abstract}

Keywords Papillary serous cystadenoma - Borderline tumor - Tumor recurrence $\cdot$ Incision site implantation

\section{Introduction}

Our patient is a 75-year-old African American female who presented for repeat repair of a symptomatic ventral hernia by a general surgeon. The hernia had been causing her periods of pain and increasing in size over several months. She had a remote history of a previous midline laparotomy, and hysterectomy years previously, for reasons she could no longer recall.

T. C. Williams $(\bowtie)$

Department of Obstetrics and Gynecology,

3901 Woodland Terrace,

West Bloomfield, MI 48323, USA

e-mail: DrTroyWilliams@hotmail.com

Tel.: +1-248-3601453

Fax: +1-248-8495469

\section{The Operation}

The patient was taken to the operating room for an elective ventral herniorrhaphy. A midline abdominal incision was made from below the xyphoid process to the umbilicus and dissection was then carried down through the subcutaneous tissue to the level of the hernia sac. The hernia sac was then dissected from adjacent tissue at the level of the fascia. The sac was then opened in the dorsal and ventral positions. A suction device was inserted into the peritoneal cavity removing 3-4 1 of brownish green fluid. The hernia sac was examined and there were no intestines present near it or the incision. The sac was removed and sent to pathology for histologic analysis. The abdomen was irrigated with saline and a careful exploration with lysis of adhesions was conducted, which revealed no perforated bowel. Upon entering the lesser sac of the abdominal cavity, there was a cauliflower-like mass confined to the tail of the pancreas and the spleen. The report from the frozen section sent to pathology returned as a probable papillary serous tumor. The decision was made to perform a distal pancreatectomy, splenectomy, and finally a ventral herniorrhaphy. The patient tolerated the procedure well and remained in stable condition throughout recovery.

\section{Radiology and pathology}

Through a radiologic search the patient was found to have been diagnosed with a large complex cystic mass with septations and solid components in the pelvic region on a computed tomography scan of the abdomen and pelvis 2 years previously (Fig. 1). At that time there was ascitic fluid extending from the pubic symphysis to the dome of the diaphragm and a moderate-sized ventral hernia present. Further investigation revealed an operative report by a gynecologic oncologist who had performed her original operation 2 years earlier. He had executed an exploratory laparotomy, drainage of 101 of ascites, total abdominal hysterectomy with bilateral salpingo-oophorectomy, pelvic and para-aortic lymphadenectomy, and ventral herniorrha- 


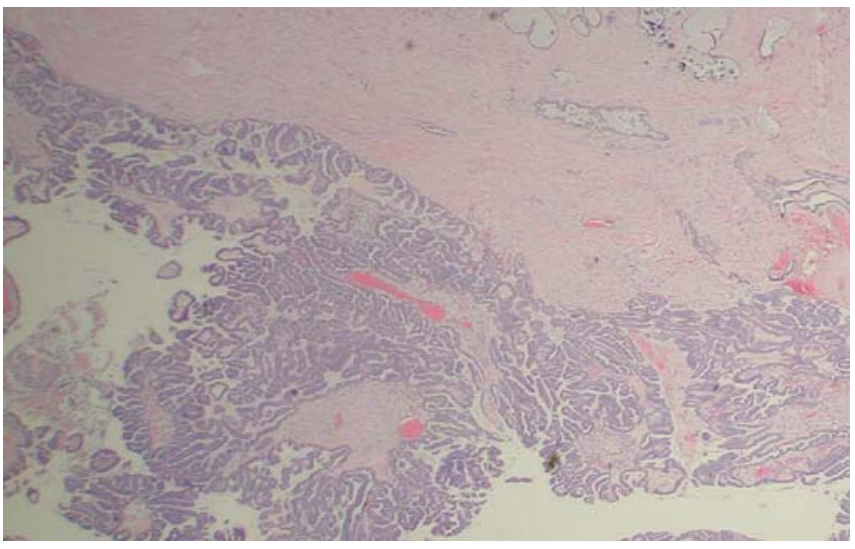

Fig. 1 Fibrous stroma $(\times 40)$, occasionally in nests, without evidence ofinfiltration. This is consistent with the diagnosis of a lowmalignancy potential tumor/borderline tumor

phy with the working diagnosis of ovarian cancer, despite a normal CA-125 tumor marker level.

The final pathology report from our specimen compared it to that submitted by the previous surgeon. The diagnosis was recurrent, non-invasive implants of borderline papillary serous cystadenoma (carcinoma of low malignant potential) evidenced in the samples from the hernia sac, the spleen, and the distal pancreas (Fig. 2). All of the specimens displayed recurrent serous papillary neoplasm with complex papillary as well as focal tubular and cystic growth. Some of the tumor nodules were associated with desmoplastic stroma at the peritoneum and spleen. Nuclear atypia ranged from mild to moderate and mitotic activity was variable, but focally present (Figs. 3,4 ). The recurrent papillary neoplasm appeared similar to that involving both ovaries previously. In consultation with the Department of Pathology at Massachusetts General Hospital, the conclusion was reached that despite the impressive morphology the recurrent neoplasm is considered to be categorized as a serous borderline tumor and only careful follow-up was suggested.

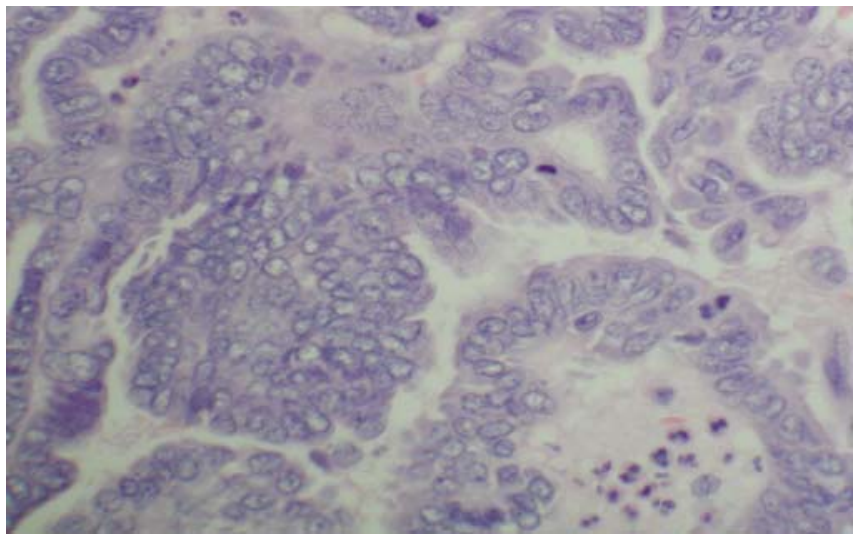

Fig. 2 Enlarged view of the specimen with complex papillary growth that is non-anaplastic.Note the mitotic activity and evidence of mitotic figures

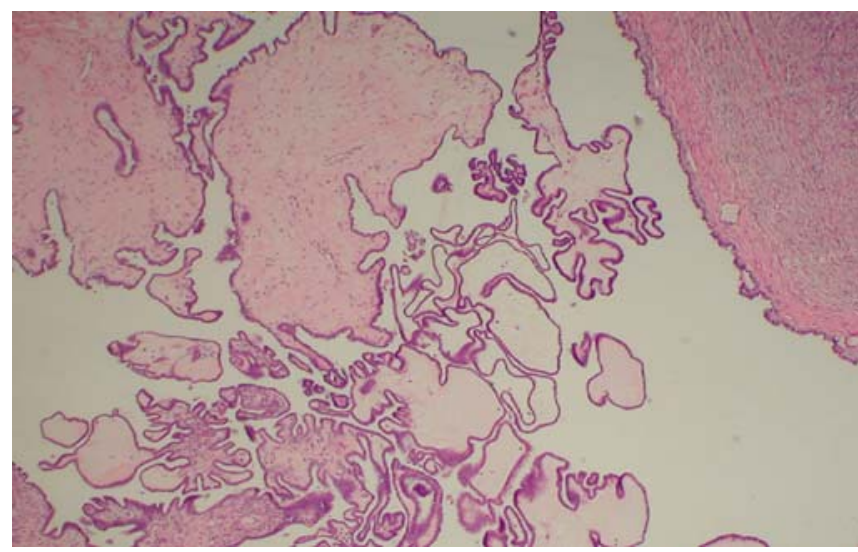

Fig. 3 Example of original tumor pathology from 2 years previously withsimilar non-invasive characteristics

\section{Discussion}

An ovarian papillary serous cystadenocarcinoma is defined as a neoplasm derived from the surface epithelium of the ovary. It is classified as papillary due to the papillary-like projections at its borders. The name serous indicates that its cells resemble those of the fallopian tubes $[1,2]$. Serous borderline tumors (SBT) are typically cystic or multicystic masses with intracystic papillary excrescences. SBT more often involve both ovaries compared with mucinous-type borderline tumors [3]. Ovarian epithelial tumors are uniquely categorized as benign (adenoma), malignant (adenocarcinoma), or of intermediate nature (borderline malignant adenocarcinoma/tumors of low malignant potential). These "intermediate tumors" have remained a source of controversy with regard to staging, prognosis, and treatment [4].

Our patient originally presented with stage IC disease, meaning that the tumor involved both ovaries and ascites were present containing malignant cells. At the time of recurrence, our patient could have met criteria to originally be classified as having stage IIIC disease because there were abdominal implants greater than $2 \mathrm{~cm}$ in diameter present in distant organs within the peritoneal cavity, but not involving the liver [4].

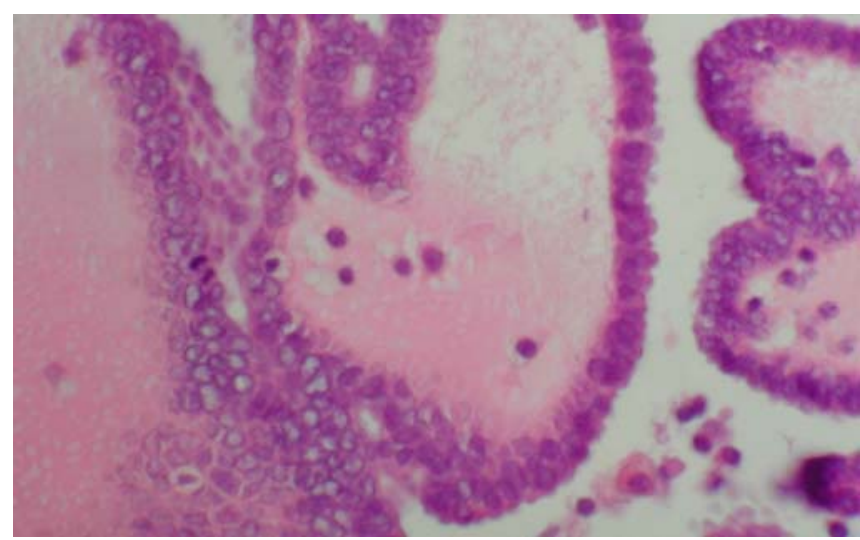

Fig. 4 Comparison slide from original tumor from 2 years previouslywith mitotic activity as noted in pathology of recurrent tumor 
The overall recurrence rate of the disease has been documented as $4.6 \%$, compared with $2.1 \%$ for stage I disease, $7.1 \%$ for stage II disease, and $14.4 \%$ for stages III and IV of the disease [5]. Our patient would be classified as having stage I disease, making recurrence in the operative setting 2 years later rather unusual. Presentation as an incisional hernia has only been documented once and that was in a patient with advanced disease. Serous borderline ovarian tumors have also been documented on rare occasions to recur at laparoscopic port sites, thus tumor seeding at wound edges remains a concern [6].

Treatment of these tumors can be accomplished by a primary conservative surgery consisting of a unilateral salpingo-oophorectomy for young women wishing to retain their potential fertility or by a complete hysterectomy with bilateral salpingo-oophorectomy $[3,7,8,9,10]$. Some authors suggest omentectomy as well as peritoneal washings to increase staging capability [11]. Cystectomy alone puts patients at an increased risk of recurrence $[10,12]$. Several studies evaluating treatment have indicated that there is no advantage to adjuvant radiation or chemotherapy compared with no post-operative treatment for borderline ovarian tumors [5]. After diagnosis was made at the time of operation, patients with stage III and stage IV disease have historically been treated with chemotherapy using platinum and alkylating agents, followed by second-look operations to evaluate the response to the chemotherapeutic agents. The role of second-look operations and tumor debulking months to years after the primary treatment is still highly debated because a large percentage of patients go on to develop recurrent disease years after their second-look procedure [4].

Some investigators suggest that some serous tumors with aggressive behavior, such as those with micropapillary architecture, represent a transition stage between SBTs and invasive serous ovarian carcinoma, even though no stromal invasion is present $[7,13]$. Several studies have noted the recurrence of borderline tumors as low-grade serous carcinoma to be an adverse prognostic feature [14]. Invasive peritoneal implants from ovarian tumors of "low malignancy potential" (LMP) behave similarly to ovarian invasive epithelial serous tumors [3, 15]. Some reports revealed no difference in survival between patients with non-invasive versus invasive peritoneal implants [3]. Thus, the borderline at which we may decide to treat SBT as adenocarcinoma needs to be explored further.

\section{Conclusion}

In our efforts to treat patients with designated serous borderline ovarian tumors, we may someday come to classify them more specifically, considering that their behavior pattern can be quite complex. Our case did not follow the $2.1 \%$ recurrence standard for stage I disease despite a thorough exploration and resection for oncologic staging. Due to the aggressive nature of the neoplasm at the time of recurrence, she required clinical and radiologic follow-up similar to that of a patient diagnosed with stage III disease or greater. In essence, she underwent a second exploratory laparotomy with tumor debulking.

A large-scale randomized prospective study comparing patients who present with stage IC disease or above and receive no adjuvant treatment with similarly staged patients receiving adjuvant therapy is needed to further delineate whether or not patients with "intermediate tumors" will benefit from more aggressive management. As of now, the only therapy being offered to many patients with serous borderline tumors is clinical follow-up after their operative diagnosis and staging.

\section{References}

1. Julian CG, Woodruff JD (1972) The biologic behavior of lowgrade papillary serous carcinoma of the ovary. Obstet Gynecol 40:860-867

2. Tornos C, Silva EG (1994) Pathology of epithelial ovarian cancer. Obstet Gynecol Clin North Am 21:63-77

3. Elchalal U, Dgani R, Piura B (1995) Current concepts in management of epithelial ovarian tumors of low malignant potential. Obstet Gynecol Surv 50:62-70

4. Mishell DR, Stenchever MA, Droegemueller W et al (1997) Neoplastic diseases of the ovary. In: Comprehensive gynecology, 3rd edn. Mosby, St. Louis, pp 901-944

5. Addar MH (1999) Recurrent stage I borderline serous ovarian tumor. Ann Saudi Med 19:243-244

6. Morrice P, Camatte S, Larregain-Fournier D (2004) Port-site implantation after laparoscopic treatment of borderline ovarian tumors. Obstet Gynecol 104:1167-1170

7. Parker RL, Clement PB, Chercover DJ (2004) Early recurrence of ovarian serous borderline tumor as high-grade carcinoma. Int J Gynecol Pathol 23:265-272

8. Mettler L, Semm K, Shive K (1997) Endoscopic management of adnexal masses. JSLS 1:103-112

9. Acs G (2002) Intra-operative consultation in gynecologic pathology. Semin Diagn Pathol 19:237-254

10. Curtin JP (1994) Management of adnexal masses. Gynecol Oncol 55:S42-S46

11. Trope CG, Kristensen G, Makar A (2000) Surgery for borderline tumor of the ovary. Semin Surg Oncol 19:69-75

12. Morris RT, Gershenson DM, Silva EG (2000) Outcome and reproductive function after conservative surgery for borderline ovarian tumors. Obstet Gynecol 95:541-547

13. Shappell HW, Riopel MA, Smith AE (2002) Diagnostic criteria and behavior of ovarian seromucinous tumors, etc. Am J Surg Pathol 26:1529-1541

14. Crispens MA (2003) Borderline ovarian tumours: a review of the recent literature. Curr Opin Obstet Gynecol 15:39-43

15. Bell DA, Scully RE (1990) Serous borderline tumors of the peritoneum. Am J Surg Pathol 14:230 